\title{
(C) OPEN ACCESS \\ Reducing cardiac arrests in the acute admissions unit: a quality improvement journey
}

\author{
Daniel J Beckett, ${ }^{1}$ Monica Inglis, ${ }^{2}$ Sharon Oswald, ${ }^{1}$ Elaine Thomson, ${ }^{3}$ \\ Wilma Harley, ${ }^{4}$ Jennifer Wilson, ${ }^{5}$ Robert C Lloyd, ${ }^{6}$ Kevin D Rooney ${ }^{7}$
}

- Additional material is published online only. To view please visit the journal online (http://dx.doi.org/10.1136/bmjqs2012-001404)

${ }^{1}$ Department of Acute Medicine, Forth Valley Royal Hospital, Larbert, Stirlingshire, UK ${ }^{2}$ Department of Clinical Governance, Falkirk Community Hospital, Falkirk, UK ${ }^{3}$ Department of Information Services, Falkirk Community Hospital, Falkirk, UK ${ }^{4}$ Department of Palliative Care, Forth Valley Royal Hospital, Larbert, Stirlingshire, UK ${ }^{5}$ Department of Oncology, Forth Valley Royal Hospital, Larbert, Stirlingshire, UK ${ }^{6}$ Department of Performance Improvement, Institute of Healthcare Improvement, Boston, Massachusetts, USA ${ }^{7}$ Institute of Care and Practice Improvement, University of the West of Scotland, Paisley, UK

\section{Correspondence to}

Dr Daniel J Beckett, Department of Acute Medicine, Forth Valley Royal Hospital, Stirling Road, Larbert, Stirlingshire FK5 4WR, UK; daniel.beckett@nhs.net

Received 29 July 2012 Revised 4 June 2013 Accepted 21 June 2013 Published Online First 4 July 2013

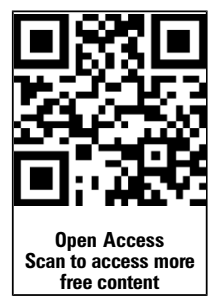

To cite: Beckett DJ, Inglis $M$, Oswald $S$, et al. BMJ Qual Saf 2013:22:1025-1031.

\begin{abstract}
Background In 2010, the acute admissions unit $(A A U)$ at Stirling Royal Infirmary had the highest number of cardiac arrests of any ward. A quality improvement project was undertaken to reduce this to $<1 / 1000$ admissions by December 2011 .

Methods In January 2011, based on initial needs assessment, we selected three initiatives to improve cardiac arrest rate: (1) structured response to deteriorating patients; (2) analysis of adverse events; and (3) improved end-of-life decision-making. We performed a failure modes effects analysis to identify reasons for the failure of early recognition and response. Ward staff conducted weekly safety meetings to engage unit staff and promote a safety culture of continuous improvement. Additionally, in July 2011 the unit adopted a ward-based clinical team structure with twice daily consultant ward rounds. Our primary outcome measure, cardiac arrests per 1000 admissions, was measured from January 2011 to August 2012.

Results Over 17 months, the number of cardiac arrests per 1000 admissions fell from a baseline of 2.8/1000 admissions to $0.8 / 1000$ admissions (71\% reduction), referrals to palliative care increased by 22 to $37 / 1000$ admissions per month (68\% increase) and the 30-day mortality of patients admitted to the AAU fell from $6.3 \%$ to $4.8 \%$ ( $24 \%$ relative reduction).
\end{abstract}

Conclusions Through adoption of a shared goal, application of improvement methodology including the model for improvement to test new innovations, and promotion of a safety culture in the AAU, cardiac arrests were successfully reduced to $<1 / 1000$ admissions per month with an associated significant fall in mortality. This was achieved with negligible cost.

\section{INTRODUCTION}

Over the past decade, acute medical units (AMUs) have been established in the UK for the provision of quality medical care in the first $48 \mathrm{~h}$ of hospital admission.
The AMU is the receiving ward for the majority of patients requiring admission to a medical in-patient bed from the emergency department (ED).

It is recognised that the quality of medical care provided in the first $48 \mathrm{~h}$ of admission is one of the most important determinants of patient outcome. ${ }^{1}$ The AMU can be a challenging environment in which to deliver care with quality as the primary focus. Bed pressures can be significant, with many units having significantly fewer beds than the recommended number (average daily admissions plus $\left.10 \%{ }^{1}\right)$, causing problems in dealing with surges in activity. Furthermore, patients in the AMU are, by definition, acutely unwell, with a greater degree of physiological instability and diagnostic doubt than one might expect to encounter in downstream wards.

The combination of these factors means that patients in the AMU are potentially at greater risk of being transferred to a critical care setting, or unexpected death (cardiac arrest), than patients in downstream wards. Two separate reports from the National Confidential Enquiry into Patient Outcomes and Deaths (NCEPOD) highlighted that physiological instability is a common precursor to admission to critical care $^{2}$ and cardiac arrest. ${ }^{3}$ In addition to this, there is evidence that cardiopulmonary resuscitation attempts are undertaken on patients with either a 'do not attempt cardio-pulmonary resuscitation' (DNACPR) order or a plan limiting future medical intervention in place, suggesting sub-optimal end-of-life care. ${ }^{3} 4$ Thus, early recognition and response to physiological instability in the AMU, along with improvements in end-of-life care, should result in a reduction in adverse events, including cardiac arrests. 
Historically, the acute admissions unit (AAU) in Stirling Royal Infirmary (SRI) had the highest number of 2222 calls of all wards ( 2222 calls-to summon the cardiac arrest team-were not made solely for cardiac arrests, but also in any circumstance where staff felt they required immediate assistance). Through a newly adopted morbidity and mortality review process, it also became clear that a significant proportion of these patients could have been considered to have received sub-optimal care. Furthermore, unsuccessful resuscitation attempts were being undertaken on patients for whom, with the benefit of hindsight, a DNACPR order would have been more appropriate.

Given these factors, a quality improvement (QI) project was undertaken with the aim of reducing the cardiac arrest rate in the AAU at SRI (and subsequently Forth Valley Royal Hospital, FVRH) to less than 1 per 1000 admissions by December 2011 (from a baseline of 2.8/1000 admissions). A cardiac arrest was defined as a loss of cardiac output where chest compressions or defibrillation was undertaken by the hospital-based resuscitation team (in line with the definition used by the National Cardiac Arrest Audit, $\mathrm{NCAA}^{5}$ ).

This work was undertaken with the support of the Scottish Patient Safety Programme (SPSP), a national QI project aiming for a $20 \%$ reduction in the hospital standardised mortality ratio (HSMR) by December $2014 .^{6}$

\section{METHODS}

The AAU at FVRH is a 46-bed unit admitting both general medical and general surgical patients. It opened in July 2011, replacing the similarly sized AAU at SRI. All admissions to the AAU are emergency admissions. These can come from several sources, including the ED (65\%), the clinical assessment unit (CAU), a 16-trolley assessment area for referrals for emergency admission from primary care $(30 \%)$ and direct admissions from the community (5\%). The unit admits on average 1500 patients per month, with a mean length of stay of 0.6 days, and accommodates patients with level 0 and level 1 care requirements. ${ }^{7}$ In 2010 the first iteration of the National Early Warning System (NEWS) ${ }^{8}$ was adopted by the AAU. However, the ED did not use an early warning system (EWS), just observation charts for recording physiological data, and these did not accompany the patients into the AAU.

A multidisciplinary team of senior and junior medical staff, nursing staff, allied health professionals and healthcare managers met in December 2010 and developed a high level driver diagram, outlining the following primary drivers to reduce the frequency of cardiac arrests in the AAU:

- Early identification and rescue of deteriorating patients

- Improved learning from adverse events

- Improved end-of-life decision-making.
The team then undertook a failure modes effects analysis to identify reasons why early identification of and response to the deteriorating patient might fail. These included:

- Lack of use of any EWS in the ED

- Unreliable NEWS calculation in the AAU

- Unreliable escalation of patients triggering NEWS, with nursing staff often unsure whom to contact.

The team developed a broad change package, designed to address each of these major issues. This included a number of safety initiatives tested using the model for improvement, and summarised in table 1 .

\section{Early identification and rescue of deteriorating patients}

The team introduced NEWS charts into the ED and NEWS became a routine part of the medical handover. The nursing handover was replaced by a standardised SBAR $^{9}$ (situation background assessment recommendation) tool. The AAU nursing staff undertook a dedicated NEWS training day to improve correct calculation of NEWS, and monthly random sampling of the accuracy of NEWS charts began with nursing champions collecting the data, to promote ownership of the project.

The team developed 'Recognition' and 'Response' checklist stickers using the model for improvement ${ }^{10}$ and multiple plan - do-study-act (PDSA) cycles. Together these comprise a structured response to the deteriorating patient. When a patient triggers an EWS of 4 or above, the ward nurse is required to place a 'Recognition' checklist sticker in the notes. This records the time of deterioration and ensures that the nurse in charge plus the relevant clinician is informed. The responding clinician is then responsible for reviewing the patient and completing a 'Response' checklist in the notes. The 'Response' checklist ensures the following:

- A documented management plan with a timeframe for further patient review

Table 1 Timeline of safety initiatives tested as part of the change package

Month Intervention

December 2010 New DNACPR protocol

December 2010 Testing new whiteboard for referral to palliative care

January 2011 Weekly AAU safety meetings tested

January 2011 Recognition and response checklist stickers tested in the AAU

February 2011 Cardiac arrest data displayed on a public QI dashboard outside the AAU

March 2011 AAU cardiac arrest data available and updated monthly on hospital intranet

June 2011 AAU cardiac arrest rate included on hospital board quality indicator dashboard

July $2011 \quad$ Move to ward-based clinical team at new site

AAU, acute admissions unit; DNACPR, do not attempt cardio-pulmonary resuscitation; QI, quality improvement. 
- Consideration of sepsis as a cause of the patient's deterioration

- Documented ceiling of care if applicable and whether or not cardio-pulmonary resuscitation would be appropriate in the event of cardiac arrest.

The aim of these checklist stickers is to act as a forcing function to improve documentation of the actions taken, ceiling of treatment and resuscitation status all within an hour of a patient triggering an EWS of 4 or above.

\section{Improved learning from adverse events}

In order to improve learning from adverse events, and to continue to foster a safety culture within the AAU, multidisciplinary safety meetings were tested. These proved popular with staff and were rapidly established on a weekly basis. All staff members (including the on-call medical staff, nursing staff, advanced nurse practitioners (ANPs), ward pharmacists, allied health professionals and medical/nursing students) were encouraged to participate, voice concerns and suggest potential solutions. A logbook was created in which staff could record the details of any event that they felt warranted discussion; in addition, all cardiac arrests and transfers to critical care were discussed. Learning points and actions were noted for discussion at the departmental monthly morbidity and mortality meeting. The Acute Medicine morbidity and mortality meetings were formalised with structured recording of adverse events and actions. Themes generated from the morbidity and mortality meetings are recorded on the departmental risk register, which feeds into the organisational risk register. This provides a direct mechanism for concerns raised at the AAU safety meeting, if of sufficient gravity, to be escalated onto the organisational risk register.

The safety meeting also proved to be a useful forum for sharing new initiatives, and also feedback of AAU data with staff. Nonetheless, it remained a challenge to communicate with every full time member of the nursing staff due to the nature of shift work. An information screen was developed, with the primary aim of sharing safety initiatives with all staff in the AAU, $24 \mathrm{~h}$ per day. It was soon adapted to also display quality indicator data, including unit process and outcome measures. Subsequently, all computer screensavers in the AAU were adapted to display the same information, ensuring maximal awareness and transparency. Finally, our process and outcome data were made available to staff, patients and family members on the newly established AAU QI dashboard, situated in the relatives' waiting room. Since February 2011, monthly cardiac arrest data have been on display to the public on our QI dashboard, with an electronic version updated on the NHS Forth Valley intranet since March 2011. Furthermore, since June 2011 the cardiac arrest rate in the AAU has been submitted to the board as an integral part of their quality dashboard.

\section{Improved end-of-life decision making}

The increasing, and earlier, identification of deteriorating patients, for whom escalation of care beyond ward level was deemed inappropriate, revealed a hitherto largely unmet need for palliative care services in the AAU. A new method of referral to palliative care was tested, referring via a whiteboard every morning, with daily visits to the AAU by the oncology/palliative care specialist nurses. Furthermore, a new policy of DNACPR was introduced in December 2010 to replace the previous 'do not attempt resuscitation' policy, making clear the distinction between cardiopulmonary resuscitation and other medical treatment.

In July 2011, all acute in-patient services within NHS Forth Valley were relocated from SRI to the newly built FVRH. At the time of transition, there was a whole system change from a traditional teambased structure to ward-based clinical teams. Historically, patients in the AAU were cared for by consultant medical staff, junior medical staff (FY1 doctors) and ANPs who also had responsibility for patients elsewhere in the hospital. Following the move to the ward-based clinical team, the staffing levels in the AAU did not change, but the ANPs, FY1 doctors and consultant acute physicians only had responsibility for patients on the AAU. Furthermore, a robust structure of twice daily consultant-led ward rounds of all patients in the AAU, in accordance with the Society for Acute Medicine and Royal College of Physicians guidelines, ${ }^{1}$ was implemented. Importantly, the size of the AAU did not change with the move of hospital, with the same bed complement, but there was a significant change in the way care was delivered.

Prior to the move, patients were routinely bedded down overnight and discharged directly from the $\mathrm{CAU}$, the trolleyed assessment area for patients referred for admission by their general practitioner. Following the move to the new site, the majority of these patients are admitted to the AAU from the CAU (which now closes overnight), resulting in a greater number of admissions to the AAU.

\section{MEASURES}

Admissions to the AAU or CAU were captured using EWARD, the NHS Forth Valley bespoke in-patient management system. Patients admitted twice or more within a month were recorded as separate episodes. Mortality was calculated at 30 days from the deaths of all patients admitted through the AAU whether still in hospital or in the community at the time of death. This was established by linkage to the National Records of Scotland (NRS) death records.

Statistical process control (SPC) methods were used to determine if significant shifts in the measures over time had occurred as a result of the interventions. The 
two control charts most appropriate for the identified measures were: the u-chart, which is the appropriate statistical tool for evaluating rate-based measures, and the p-chart, which is the chart of choice when measuring percentages or proportions. ${ }^{11}$ The SPC charts were utilised to assess the impact of the improvement project on both cardiac arrests and all 2222 calls within the AAU. Reliable baseline data were only available for number of cardiac arrests from August 2010 and therefore the 6-month period from August 2010 to the first intervention in January 2011 was used as the baseline.

The outcome measures that were recorded included:

- The cardiac arrest rate (number of cardiac arrests in the AAU per 1000 admissions per month)

- The 2222 call rate (number of 2222 calls in the AAU per 1000 admissions per month)

- The 30-day mortality of patients admitted through the AAU

- The 30-day mortality of all patients admitted to the AAU or CAU.

The process measures that were recorded during this improvement project included:

- Percentage accuracy of EWS recording as measured by review of 20 randomly sampled charts per month

- Percentage of patients triggering EWS of 4 or above having completed recognition and response checklists in case notes as measured by review of 20 randomly sampled charts per month
- Number of people attending the weekly multidisciplinary safety meeting

- Percentage of cardiac arrest audit forms being completed correctly

- Number of patients referred to the oncology liaison/ palliative care services from the AAU.

The balancing measures that were monitored included:

- Number of transfers from the AAU to critical care

- Number of cardiac arrests outside the AAU.

\section{RESULTS}

Immediately following the mandatory NEWS workshop for nursing staff in the AAU, the accuracy of NEWS recording per month improved from $65 \%$ to $100 \%$ and has demonstrated sustained improvement at $>90 \%$ subsequently.

The recognition checklist was completed more reliably with a mean of $93 \%$ correctly completed each month. The response checklist was less reliable, with a mean of $73 \%$ correctly completed each month.

With the changes to the new ward-based team structure, coincident with the unit move, there was significantly greater throughput of patients in the AAU, largely due to patients no longer needing to be bedded in the CAU. Mean monthly admissions to the AAU increased from 1002 to 1533 (53\%). There was an associated change in the AAU average length of stay from 1.2 to 0.6 days, a fall of $50 \%$.

Following implementation of the safety initiatives, the number of cardiac arrests per 1000 admissions fell

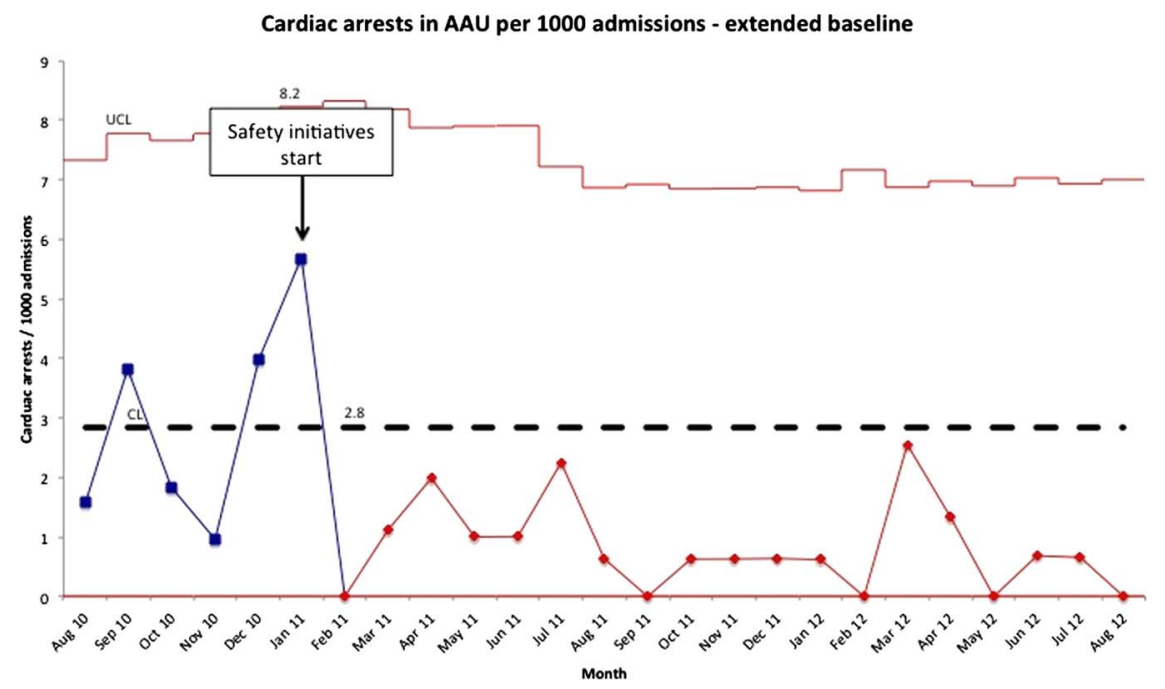

Figure 1 U-chart demonstrating cardiac arrests in the acute admissions unit (AAU) per 1000 admissions. Following the implementation of safety initiatives in January 2011, a significant downward shift was immediately observed in the number of cardiac arrests with 19 consecutive points below the baseline mean. Segmentation of the data following the significant shift in cardiac arrests demonstrates a fall from mean of 2.8 (baseline) per month to 0.8 per month (February 2011-August 2012), a relative reduction of $71 \%$ in cardiac arrests. In interpreting statistical process control charts, several things should be noted. (1) Each chart is shown with the confidence limits and centre line frozen from the baseline (August 2010-January 2011). Any significant shifts (defined as eight consecutive data points above or below the mean or centre line on the chart) or trends (defined as six or more consecutive data points constantly increasing or decreasing) in the data thereafter are highlighted. If a significant shift is detected then the data are rephased from this point and the new mean compared with the baseline mean. (2) Since balancing measures are employed to ensure that as one measure improves there is not a concomitant negative movement in other key variables, the balancing measures are not expected to demonstrate either shifts or trends. CL, centre line; UCL, upper control limit. 
from 2.8 to 0.8 per month-a reduction of $71 \%$ (see figure 1). There was a $73 \%$ reduction in all 2222 calls from the AAU from 4.9/1000 admissions to 1.3/1000 admissions (see figure 2). There was no significant change in the number of 2222 calls occurring outside the AAU, including in intensive care (see online supplementary appendix figure S1), but there was a significant increase (relative increase 68\%; see online supplementary appendix figure S2) in the number of palliative care referrals from the AAU.

There was a significant fall in the 30-day mortality of patients admitted to the AAU from $6.3 \%$ to $4.8 \%$ (relative reduction of $24 \%$; see figure 3), suggesting the reduction in cardiac arrests was achieved through a combination of improved palliative care and improved rescue of the deteriorating patient. The 30-day mortality of all patients attending the AAU or CAU was calculated and also demonstrated a significant fall, from $5.8 \%$ to $4.4 \%$ (a relative reduction of 24\%; see online supplementary appendix figure S3).

Over the period of this project, the HSMR also fell, from 1.10 (October-December 2010) to 0.88 (AprilJune 2012), a relative reduction of $20 \%$.

\section{DISCUSSION}

Over an 18-month period, cardiac arrests in the AAU at SRI and latterly FVRH were reduced from 2.8 to 0.8 per 1000 admissions. There was also a fall in all 2222 calls in the AAU, and an increase in referrals to palliative care. There was a significant fall (relative reduction of 24\%) in 30-day mortality in patients admitted to the AAU (or the AAU/CAU) over this time period.

Key to this success has been the development of a structured response to the deteriorating patient. Following the implementation of reliable EWS across the AAU and ED, and the recognition and response checklists, plus weekly safety meetings in the AAU at SRI, there was an immediate fall in the number of cardiac arrests, which was sustained thereafter. However, a reduction in all 2222 calls was not observed until several months later, immediately before the move to the new site and ward-based clinical team. It is likely that that the checklists, through standardising the response to the deteriorating patient, were responsible for the reduction in cardiac arrests. The key to this was earlier identification of patients at risk of deterioration, allowing the clinical team, in discussion with patients and relatives, to make decisions early in the admission regarding the appropriateness of escalation of therapy, and resuscitation status. However, nursing staff prior to the move still used 2222 calls to summon immediate help. This method of communication and escalation of care became largely redundant with the dedicated wardbased clinical team on the new hospital site. This new way of working was not dependent on the site move, which rather was utilised as an opportunity to change what had previously been perceived to be an inferior model of patient care. To tease out the relative contribution of each intervention to the reduction in mortality and 2222 calls per month would require a planned experimentation approach. ${ }^{12}$ Finally, along with the reduction in the number of cardiac arrests and 2222 calls per month, there was also reduction in month-to-month variability, indicating greater system stability.

During the project, there was scepticism from some medical staff who suggested that the reduction in cardiac arrests was achieved largely through earlier DNACPR decisions being taken (which was, of

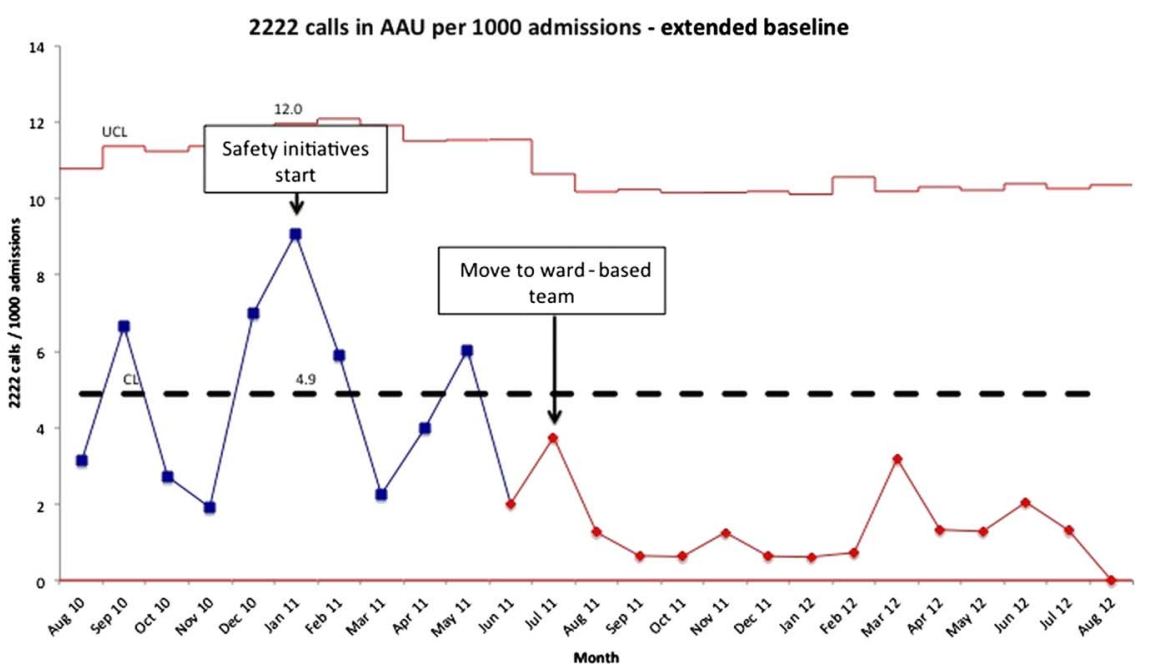

Figure 2 U-chart demonstrating all 2222 calls in the acute admissions unit (AAU) per 1000 admissions. Following the implementation of safety initiatives in January 2011, a significant downward shift in the number of 2222 calls was observed after a period of 5 months. Starting in June 2011, 15 consecutive points are charted below the baseline mean. Segmentation of the data following the significant shift in all 2222 calls demonstrates a fall from a mean of 4.9 per month (baseline) to 1.3 per month (June 2011-August 2012), a relative reduction of $73 \%$. See figure 1 caption for advice on interpreting the chart. $C L$, centre line; $U C L$, upper control limit. 


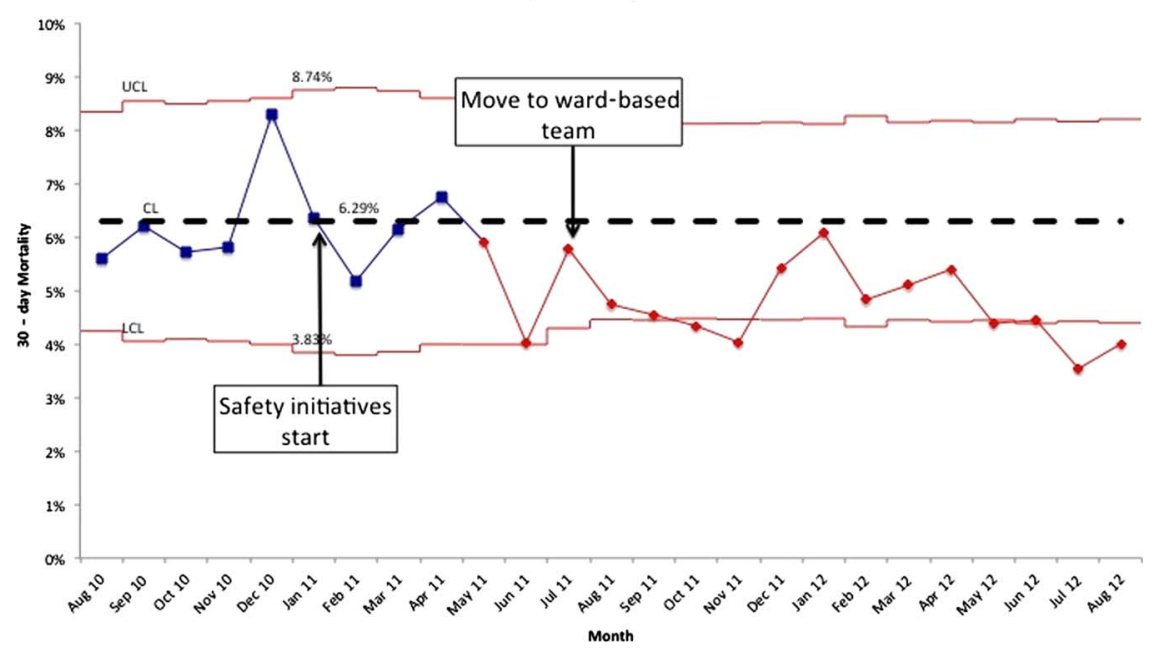

Figure 3 P-chart with extended baseline for 30-day mortality of patients admitted to the acute admissions unit (AAU). A significant downwards shift in 30-day mortality is observed from May 2011, 4 months after implementation of the safety initiatives, and 2 months prior to the move to the new ward-based system. Segmentation of the data following the significant shift in 30-day mortality demonstrates a fall from 6.3\% per month (baseline) to $4.8 \%$ per month (May 2011-August 2012), a relative reduction of $24 \%$. See figure 1 caption for advice on interpreting the chart. $C L$, centre line; $L C L$, lower control limit; $U C L$, upper control limit.

course, one of the primary drivers). However, if the reduction in cardiac arrests was due solely to earlier decisions being made to limit treatment and implement DNACPR decisions, then there should have been no impact on 30-day mortality. The significant fall in mortality 4 months after the implementation of the AAU safety initiatives, suggests that the observed reduction in cardiac arrests was not due solely to earlier, appropriate DNACPR decisions, but also to improvements in recognition of and response to deteriorating patients. Other sceptics suggested that the reduction in cardiac arrests in the AAU was due to patients being moved out of the AAU early and sustaining a cardiac arrest elsewhere. The use of monthly cardiac arrest calls outside the AAU (including in the intensive care unit) as a balancing measure demonstrated that this simply was not the case.

When interpreting cardiac arrest and mortality rates, it is important to recognise changes in AAU activity at the time of site move. A greater number of patients are now admitted to the AAU who were previously bedded down and directly discharged from the CAU. This increase in the number of admissions increases the denominator in per 1000 admission calculations. Furthermore, these patients, previously discharged directly from the CAU but now admitted to the AAU, might be considered 'lower risk' and thus their subsequent inclusion in AAU figures after the move to the new hospital might artificially reduce AAU mortality without representing a genuine improvement in outcome. In order to eliminate this potential bias, the 30-day mortality of all patients admitted to the AAU or CAU was calculated. This displayed a fall of similar magnitude, suggesting that the observed reduction in mortality is real, rather than due to bias. Finally, there was no change in the catchment population with the move of hospital site.

The majority of emergency admissions to FVRH are admitted through the AAU, and it is therefore probable that the process improvements in the AAU are likely to have contributed significantly to the reduction in HSMR observed over the duration of the project. Other factors which may well have also contributed include enhanced community palliative care services, more accurate diagnostic coding and increasing focus on patient safety on downstream wards, all of which were targeted for improvement at some point during this project.

Our hospital does not utilise a medical emergency team (MET), but has a traditional cardiac arrest team that responds to all 2222 calls including those for 'periarrest' patients. In planning this project, thought was given to the establishment of a MET, but after consideration of the recent NICE guideline, ${ }^{13}$ Cochrane review ${ }^{14}$ and Resuscitation Council guideline ${ }^{15}$ none of which recommended implementation of a MET, we decided to improve the reliability of escalation through the patient's own clinical team (nursing and medical staff), through development of a structured response to the deteriorating patient. This study suggests that this approach can improve patient outcomes while retaining a focus on patient-centred care.

This project was carried out with the engagement of the AAU multidisciplinary team; no additional staffing resources were available (or necessary). The costs of the project have been negligible, for example, the recognition and response checklists are still printed in-house in the AAU by the ward clerkess.

Following the success of this project, the recognition and response checklists are being tested in other 
AMUs across Scotland. Additionally, they are being adopted by the Scottish Patient Safety Program to form the basis of a national, structured approach to the deteriorating patient.

\section{CONCLUSIONS}

The introduction of a number of key safety interventions including the development of a structured response to the deteriorating patient, weekly safety meetings and a ward-based clinical team in the AAU at SRI and latterly FVRH, led to a sustained reduction in cardiac arrests and 2222 calls, accompanied by a significant fall in 30-day mortality. Greater engagement with palliative care reflects the movement to a patientcentred approach. The public display of our cardiac arrest rates has helped change the culture in our unit to one of transparency and safety. Importantly, all the interventions tested here to deliver person-centred care and foster a safety culture, have been achieved in a cost neutral manner and can be tested in any hospital in the world and adapted to local context.

Correction notice This paper has been corrected since it was published online first. The expansions of CL, UCL and LCL have been corrected in the figure captions.

Acknowledgements We thank Dr Iain Wallace (Medical Director, NHS Forth Valley), Ian Aitken (General Manager, NHS Forth Valley), Michael Mertens (Kaiser Permanente Healthcare Performance Improvement), Jane Roessner (Institute for Healthcare Improvement) and The Forth Valley Royal Hospital AAU multidisciplinary team.

Contributors DJB was the clinical lead for the project, wrote the manuscript and is the guarantor. MI provided project support and reviewed the manuscript. SO provided clinical support for the project and reviewed the manuscript. ET performed data analysis. WH and JW contributed to acquisition of data. RCL and KDR provided expert advice and reviewed the manuscript.

\section{Competing interests None.}

Provenance and peer review Not commissioned; externally peer reviewed.

Data sharing statement Data available on request from the corresponding author.

Open Access This is an Open Access article distributed in accordance with the Creative Commons Attribution Non Commercial (CC BY-NC 3.0) license, which permits others to distribute, remix, adapt, build upon this work non-commercially, and license their derivative works on different terms, provided the original work is properly cited and the use is noncommercial. See: http://creativecommons.org/licenses/by-nc/3.0/

\section{REFERENCES}

1 Acute Medicine Taskforce. Acute medical care. The right person, in the right setting-first time. Report of the Acute Medicine Task Force. London: Royal College of Physicians, 2007.

2 NCEPOD. An acute problem? http://www.ncepod.org.uk/ 2005aap.htm (accessed 8 Mar 2012).

3 NCEPOD. Time to intervene? http://www.ncepod.org.uk/ 2012report1/downloads/CAP_fullreport.pdf (accessed 20 May 2013).

4 Kause J, Smith G, Prytherch D, et al. A comparison of Antecedents to Cardiac Arrests, Deaths and Emergency Intensive care Admissions in Australia and New Zealand, and the United Kingdom-the ACADEMIA study. Resuscitation 2004;62:275-82.

5 Resuscitation Council (UK). National Cardiac Arrest Audit (NCAA). http://www.resus.org.uk/pages/ncaa.htm (accessed 8 Mar 2012).

6 Rooney KD, Leitch J. Quality and safety in NHS Scotland. Br J Diabetes Vasc Dis 2010;10:98-100.

7 Intensive Care Society. Levels of critical care for adult patients. http://www.sicsag.scot.nhs.uk/Levels_of_Care_Intensive_Care_ Society_2009.pdf (accessed 20 May 2013).

8 Royal College of Physicians. National Early Warning Score (NEWS). http://www.rcplondon.ac.uk/resources/ national-early-warning-score-news (accessed 20 May 2013).

9 Institute for Healthcare Improvement. SBAR technique for communication: a situational briefing model. http://www.ihi. org/knowledge/Pages/Tools/ SBARTechniqueforCommunicationASituationalBriefingModel. aspx (accessed 24 Feb 2012).

10 Langley GJ, Moen RD, Nolan KM, et al. The improvement guide: a practical approach to enhancing organizational performance. San Francisco, CA: Jossey-Bass, 2009.

11 Lloyd RC. Quality health care: a guide to developing and using indicators. Sudbury, MA: Jones and Bartlett, 2004.

12 Moen RD, Nolan TW, Provost LP. Quality improvement through planned experimentation. McGraw-Hill, 1999.

13 NICE. Acutely ill patients in hospital (CG50). Recognition of and response to acute illness in adults in hospital. Manchester: NICE, 2007.

14 McGaughey J, Alderice F, Fowler R, et al. Outreach and early warning systems (EWS) for the prevention of intensive care admission and death of critically ill adult patients in general hospital wards. Cochrane Database of Syst Rev 2007(3):CD005529.

15 Resuscitation Council (UK). Prevention of cardiac arrest and decisions about cardiopulmonary resuscitation. London: Resuscitation Council (UK), 2010. 